\title{
Fixed points of differences of meromorphic functions
}

\author{
Zhaojun $\mathrm{Wu}^{1,2^{*}}$ (D) and Jia $\mathrm{Wu}^{3}$
}

${ }^{*}$ Correspondence: wuzj52@hotmail.com

'school of Mathematics and Statistics, Hubei University of

Science and Technology, Xianning, China

${ }^{2}$ Hubei Key Laboratory of Applied Mathematics, Faculty of Mathematics and Statistics, Hubei University, Wuhan, China Full list of author information is available at the end of the article

\begin{abstract}
Let $f$ be a transcendental meromorphic function of finite order and $c$ be a nonzero complex number. Define $\Delta_{c} f=f(z+c)-f(z)$. The authors investigate the existence on the fixed points of $\Delta_{c} f$. The results obtained in this paper may be viewed as discrete analogues on the existing theorem on the fixed points of $f^{\prime}$. The existing theorem on the fixed points of $\Delta_{c} f$ generalizes the relevant results obtained by (Chen in Ann. Pol. Math. 109(2):153-163, 2013; Zhang and Chen in Acta Math. Sin. New Ser. 32(10):1189-1202, 2016; Cui and Yang in Acta Math. Sci. 33B(3):773-780, 2013) et al.
\end{abstract}

Keywords: Difference operator; Fixed point; Borel exceptional values; Deficiency

\section{Introduction}

Let $f(z)$ be a function meromorphic in the complex plane $C$. We use the general notation of the Nevanlinna theory (see [12, 20, 23]) such as $m(r, f), N(r, f), T(r, f), m\left(r, \frac{1}{f-a}\right)$, $N\left(r, \frac{1}{f-a}\right), \ldots$, and assume that the reader is familiar with these notations. We also use $S(r, f)$ to denote any quantity of $S(r, f)=o(T(r, f))(r \rightarrow \infty)$, possibly outside a set with finite logarithmic measure. The order and the lower order of $f(z)$ are denoted by $\sigma(f)$ and $\mu(f)$ respectively.

For any $a \in C$, the exponent of convergence of zeros of $f(z)-a$ (or poles of $f(z)$ ) is denoted by $\lambda(f, a)$ (or $\lambda\left(\frac{1}{f}\right)$ ). Especially, we denote $\lambda(f, 0)$ by $\lambda(f)$. If $\lambda(f, a)<\sigma(f)$ (or $\lambda\left(\frac{1}{f}\right)<\sigma(f)$ ), then $a$ (or $\infty$ ) is said to be a Borel exceptional value of $f(z)$. Nevanlinna's deficiency of $f$ with respect to complex number $a \in C \cup\{\infty\}$ is defined by

$$
\delta(a, f)=\liminf _{r \rightarrow \infty} \frac{m\left(r, \frac{1}{f-a}\right)}{T(r, f)}=1-\limsup _{r \rightarrow \infty} \frac{N\left(r, \frac{1}{f-a}\right)}{T(r, f)} .
$$

If $a=\infty$, then one should replace $N\left(r, \frac{1}{f-a}\right)$ in the above formula by $N(r, f)$.

A point $z_{0} \in C \cup\{\infty\}$ is said to be a fixed point of $f(z)$ if $f\left(z_{0}\right)=z_{0}$. There is a considerable number of results on the fixed points of meromorphic functions, we refer the reader to Chuang and Yang [7]. It follows Chen and Shon [2, 4], we use the notation $\tau(f)$ to denote the exponent of convergence of fixed points of $f$, i.e.,

$$
\tau(f)=\limsup _{r \rightarrow \infty} \frac{\log N\left(r, \frac{1}{f-z}\right)}{\log r} .
$$

In 1993, Lahiri [13] proved the following theorem.

\section{Springer}

(c) The Author(s) 2019. This article is distributed under the terms of the Creative Commons Attribution 4.0 International License (http://creativecommons.org/licenses/by/4.0/), which permits unrestricted use, distribution, and reproduction in any medium, provided you give appropriate credit to the original author(s) and the source, provide a link to the Creative Commons license, and indicate if changes were made. 
Theorem A Let $f$ be a transcendental meromorphic function in the plane. Suppose that there exists $a \in C$ with $\delta(a, f)>0$ and $\delta(\infty, f)=1$. Then $f$ has infinitely many fixed points.

In this paper, we shall study the fixed points of the differences of meromorphic functions. For each $c \in C \backslash\{0\}$, the forward difference $\Delta_{c}^{k+1} f(z)$ is defined (see [1]) by

$$
\Delta_{c} f(z)=f(z+c)-f(z), \Delta_{c}^{2} f(z)=\Delta_{c} f(z+c)-\Delta_{c} f(z) .
$$

Especially, we denote $\Delta_{1} f(z)$ by $\Delta f(z)$.

Recently, some well-known facts of the Nevanlinna theory have been extended for the differences of meromorphic functions (see [5, 6, 9-11, 14-18]). For the existence on the fixed points of differences, Cui and Yang [8] have proved the following theorems.

Theorem B ([8]) Let $f$ be a function transcendental and meromorphic in the plane with the order $\sigma(f)=1$. If $f$ has finitely many poles and infinitely many zeros with exponent of convergence of zeros $\lambda(f) \neq 1$, then $\Delta f$ has infinitely many zeros and fixed points.

Theorem C ([8]) Letf be a non-periodic function transcendental and meromorphic in the plane with the order $\sigma(f)=1, \max \left\{\lambda(f), \lambda\left(\frac{1}{f}\right)\right\} \neq 1$. If $f$ has infinitely many zeros, then $\Delta f$ has infinitely many zeros and fixed points.

The conditions of Theorems $B$ and $C$ imply that $0, \infty$ are Borel exceptional values. If $\infty$ and $d \in C$ are Borel exceptional values of $f$, Chen [3] obtains the following theorem.

Theorem $\mathbf{D}([3])$ Let $f$ be a finite order meromorphic function such that $\lambda\left(\frac{1}{f}\right)<\sigma(f)$, and let $c \in C \backslash\{0\}$ be a constant such that $f(z+c) \not \equiv f(z)$. If $f(z)$ has a Borel exceptional value $d \in C$, then $\tau\left(\Delta_{c} f\right)=\sigma(f)$.

In [22], Zhang and Chen showered that the condition $\lambda\left(\frac{1}{f}\right)<\sigma(f)$ in Theorem D cannot be omitted. Moreover, they obtained the following theorem.

Theorem $\mathbf{E}$ ([22]) Let $f$ be a finite order meromorphic function, and let $c \in C \backslash\{0\}$ be a constant such that $f(z+c) \not \equiv f(z)$. If $f(z)$ has two Borel exceptional values, then $\tau\left(\Delta_{c} f\right)=$ $\sigma(f)$.

In [19], Yi and Yang have proved the following theorem.

Theorem $\mathbf{F}$ ([19]) Let $f$ be a transcendental meromorphic function in $C$ with a positive order. Iff has two distinct Borel exceptional values, say $a_{1}$ and $a_{2}$, then the order of $f$ is a positive integer or $\infty$ and $\sigma(f)=\mu(f), \delta\left(a_{1}, f\right)=\delta\left(a_{2}, f\right)=1$.

By Theorem $\mathrm{F}$, we can derive that the order of $f$ in Theorems $\mathrm{D}$ and $\mathrm{E}$ is a positive integer. Is it necessary to ask if the order of $f$ is an integer?, i.e., Can we get similar results as those in Theorems B, C, D, and $\mathrm{E}$ if the order of $f$ is not a positive integer? The main purpose of this paper is to study this question. In fact, we shall prove the following theorems.

Theorem 1.1 Let $f$ be a transcendental meromorphic function of finite order in the plane. Suppose that $c \in C \backslash\{0\}$ such that $\Delta_{c} f \not \equiv 0$. If there is $a \in C$ with $\delta(a, f)>0$ and $\delta(\infty, f)=1$, then $\Delta_{c} f$ have infinitely many fixed points and $\tau\left(\Delta_{c} f\right)=\sigma(f)$. 
Theorem 1.2 Let $f$ be a transcendental meromorphic function of finite order in the plane. Suppose that $c \in C \backslash\{0\}$ such that $\Delta_{c} f \not \equiv 0$. If $\delta(\infty, f)=1, \delta(0, f)=1$, then

$$
T\left(r, \Delta_{c} f\right) \sim T(r, f) \sim N\left(r, \frac{1}{\left(\Delta_{c} f\right)-z}\right)
$$

as $r \rightarrow \infty, r \notin E$, where $E$ is a possible exception set of $r$ with finite logarithmic measure.

Let $f(z)=\frac{e^{z}}{z}$, then $N(r, f)=\log r=S(r, f), N\left(r, \frac{1}{f}\right)=0$ and $\Delta_{c} f=\frac{\left(e^{c}-1\right) z-1}{z(z+c)} e^{z} \not \equiv 0$. By the second fundmental theorem, we have

$$
T\left(r, \Delta_{c} f\right) \sim T(r, f) \sim N\left(r, \frac{1}{\left(\Delta_{c} f\right)-z}\right) \quad(r \rightarrow \infty)
$$

and $\tau\left(\Delta_{c} f\right)=\sigma(f)$.

\section{Proof of Theorems 1.1 and 1.2}

Lemma 2.1 ([6]) Let $f(z)$ be a finite order meromorphic function, then, for each $k \in N$, $\sigma\left(\Delta_{c}^{k} f\right) \leq \sigma(f)$.

Lemma 2.2 ([9]) Let $f$ be a transcendental meromorphic function of finite order. Then, for any positive integer $n$, we have

$$
m\left(r, \frac{\Delta_{c}^{n} f(z)}{f(z)}\right)=S(r, f) .
$$

Lemma 2.3 Let $f$ be a transcendental meromorphic function of finite order. Suppose that $c \in C \backslash\{0\}$ such that $\Delta_{c} f \not \equiv 0$ and $\delta(0, f)>0$. Then $\Delta_{c} f$ is a transcendental and meromorphic function of finite order.

Proof From Lemma 2.1, we know that $\sigma\left(\Delta_{c} f\right) \leq \sigma(f)<+\infty$. If $\Delta_{c} f$ is not a transcendental meromorphic function, then there is a rational function $R(z)$ such that $R(z) \Delta_{c} f \equiv 1$, i.e.,

$$
\frac{1}{f} \equiv R(z) \frac{\Delta_{c} f}{f}
$$

Applying Lemma 2.2 and noticing that $f(z)$ is transcendental, we have

$$
m\left(r, \frac{1}{f}\right) \leq m(r, R(z))+m\left(r, \frac{\Delta_{c} f}{f}\right)=S(r, f) .
$$

This contradicts $\delta(0, f)>0$. Thus $\Delta_{f} f$ is a transcendental and meromorphic function of finite order.

Lemma 2.4 ([11]) Let $f(z)$ be a transcendental meromorphic function of finite order, then

$$
m\left(r, \frac{f(z+c)}{f}\right)=S(r, f)
$$


Lemma 2.5 $([14,21])$ Letf be a transcendental meromorphic function of finite order. Then

$$
\begin{aligned}
& N(r, f(z+c))=N(r, f)+S(r, f), \\
& T(r, f(z+c))=T(r, f)+S(r, f) .
\end{aligned}
$$

Lemma 2.6 Let $f$ be a finite order transcendental meromorphic function. Suppose that $c \in C \backslash\{0\}$ such that $\Delta_{c} f \not \equiv 0$. If $\delta(0, f)>0$, then

$$
T(r, f) \leq 4 N(r, f)+N\left(r, \frac{1}{f}\right)+N\left(r, \frac{1}{\left(\Delta_{f} f\right)-z}\right)+S(r, f) .
$$

Proof By Lemma 2.3, we know that $\Delta_{c} f$ is a transcendental meromorphic function. Put $F=\Delta_{c} f$, then there is $\eta \in C \backslash\{0\}$ such that $z \Delta_{\eta} F-\eta F(z) \not \equiv 0$. If not, then

$$
\frac{F(z)}{z} \equiv \frac{F(z+\eta)}{z+\eta}
$$

holds for any $\eta \in C \backslash\{0\}$. Hence $\frac{F(z)}{z}$ is a constant, which contradicts $F=\Delta_{c} f$ is a transcendental meromorphic function. Hence there is $\eta \in \backslash\{0\}$ such that $z \Delta_{\eta} F-\eta F(z) \not \equiv 0$, i.e.,

$$
\begin{aligned}
z \Delta_{\eta} & F-\eta F(z) \\
& =z \Delta_{\eta}\left(\Delta_{c} f\right)-\eta \Delta_{c} f \\
& =z \Delta_{\eta}\left(\left(\Delta_{c} f\right)-z\right)-\eta\left(\left(\Delta_{c} f\right)-z\right) \\
& =z f(z+c+\eta)-z f(z+\eta)-(z+\eta) f(z+c)+(z+\eta) f(z) \not \equiv 0 .
\end{aligned}
$$

Noticing

$$
\frac{1}{f}=\frac{\Delta_{c} f}{z f}-\frac{z \Delta_{\eta}\left(\Delta_{c} f\right)-\eta \Delta_{c} f}{z f} \frac{\left(\Delta_{c} f\right)-z}{z \Delta_{\eta}\left(\Delta_{c} f\right)-\eta \Delta_{c} f} .
$$

Combining (1), (2) and Lemmas 2.2, 2.4, we can get

$$
\begin{aligned}
m\left(r, \frac{1}{f}\right) & \\
\leq & m\left(r, \frac{\Delta_{c} f}{z f}\right)+m\left(r, \frac{z \Delta_{\eta}\left(\Delta_{c} f\right)-\eta \Delta_{c} f}{z f}\right) \\
& +m\left(r, \frac{\left(\Delta_{c} f\right)-z}{z \Delta_{\eta}\left(\Delta_{c} f\right)-\eta \Delta_{c} f}\right)+\log 2 \\
\leq & m\left(r, \frac{\Delta_{c} f}{f}\right)+m\left(r, \frac{f(z+c+\eta)}{f}\right)+m\left(r, \frac{f(z+c)}{f}\right) \\
& +m\left(r, \frac{f(z+\eta)}{f}\right)+m\left(r, \frac{\left(\Delta_{c} f\right)-z}{z \Delta_{\eta}\left(\Delta_{c} f\right)-\eta \Delta_{c} f}\right)+O(\log r) \\
= & m\left(r, \frac{\left(\Delta_{c} f\right)-z}{z \Delta_{\eta}\left(\Delta_{c} f\right)-\eta \Delta_{c} f}\right)+S(r, f) .
\end{aligned}
$$


Applying the first fundamental theorem of Nevanlinna theory, we have

$$
T(r, f) \leq N\left(r, \frac{1}{f}\right)+m\left(r, \frac{\left(\Delta_{c} f\right)-z}{z \Delta_{\eta}\left(\Delta_{c} f\right)-\eta \Delta_{c} f}\right)+S(r, f),
$$

and we get

$$
\begin{aligned}
& m\left(r, \frac{\left(\Delta_{c} f\right)-z}{z \Delta_{\eta}\left(\Delta_{c} f\right)-\eta \Delta_{c} f}\right) \\
& \quad \leq m\left(r, \frac{z \Delta_{\eta}\left(\Delta_{c} f\right)-\eta \Delta_{c} f}{\left(\Delta_{c} f\right)-z}\right)+N\left(r, \frac{z \Delta_{\eta}\left(\Delta_{c} f\right)-\eta \Delta_{c} f}{\left(\Delta_{c} f\right)-z}\right)+O(1) .
\end{aligned}
$$

It follows from (1) that

$$
m\left(r, \frac{z \Delta_{\eta}\left(\Delta_{f} f\right)-\eta \Delta_{c} f}{\left(\Delta_{f} f\right)-z}\right) \leq m\left(r, \frac{\Delta_{\eta}\left(\left(\Delta_{c} f\right)-z\right)}{\left(\Delta_{c} f\right)-z}\right)+S(r, f) .
$$

Applying Lemma 2.3 and Lemma 2.5, we know that $\left(\Delta_{f} f\right)-z$ is a transcendental meromorphic function of finite order and

$$
T\left(r,\left(\Delta_{c} f\right)-z\right) \leq 2 T(r, f)+S(r, f) .
$$

Therefore,

$$
S\left(r,\left(\Delta_{c} f\right)-z\right)=S(r, f) .
$$

It follows from Lemma 2.2 and (6) that

$$
m\left(r, \frac{z \Delta_{\eta}\left(\Delta_{f} f\right)-\eta \Delta_{c} f}{\left(\Delta_{c} f\right)-z}\right)=S(r, f) .
$$

By Lemma 2.5 and (1), we derive

$$
\begin{aligned}
& N\left(r, \frac{z \Delta_{\eta}\left(\Delta_{c} f\right)-\eta \Delta_{c} f}{\left(\Delta_{c} f\right)-z}\right) \\
& \quad \leq N\left(r, z \Delta_{\eta}\left(\Delta_{c} f\right)-\eta \Delta_{c} f\right)+N\left(r, \frac{1}{\left(\Delta_{c} f\right)-z}\right) \\
& \quad \leq N\left(r, \frac{1}{\left(\Delta_{f} f\right)-z}\right)+4 N(r, f)+S(r, f) .
\end{aligned}
$$

Combining (3)-(5) and (7)-(8), we have

$$
T(r, f) \leq 4 N(r, f)+N\left(r, \frac{1}{f}\right)+N\left(r, \frac{1}{\left(\Delta_{c} f\right)-z}\right)+S(r, f) .
$$




\subsection{Proof of Theorem 1.1}

Denoting $g=f-a$, by Lemma 2.6, we have

$$
\begin{aligned}
T(r, f) & =T(r, g)+O(1) \\
& \leq 4 N(r, g)+N\left(r, \frac{1}{g}\right)+N\left(r, \frac{1}{\left(\Delta_{c} g\right)-z}\right)+S(r, g) \\
& =4 N(r, f)+N\left(r, \frac{1}{f-a}\right)+N\left(r, \frac{1}{\left(\Delta_{c} f\right)-z}\right)+S(r, f) .
\end{aligned}
$$

Since $\delta(a, f)>0$ and $\delta(\infty, f)=1$, then there is a positive number $\theta<1$ such that

$$
\begin{aligned}
& N\left(r, \frac{1}{f-a}\right)<\theta T(r, f), \\
& N(r, f) \leq o(1) T(r, f) .
\end{aligned}
$$

Combining (9)-(11), we can get

$$
(1-o(1)-\theta) T(r, f) \leq N\left(r, \frac{1}{\left(\Delta_{c} f\right)-z}\right) .
$$

Note that $f$ is transcendental, we can get that $\Delta_{c} f$ has infinitely many fixed points and $\tau\left(\Delta_{c} f\right)=\sigma(f)$ from (12).

\subsection{Proof of Theorem 1.2}

Since

$$
\begin{aligned}
m\left(r, \frac{1}{f}\right) & =m\left(r, \frac{\Delta_{c} f}{f} \frac{1}{\Delta_{c} f}\right) \leq m\left(r, \frac{\Delta_{c} f}{f}\right)+m\left(r, \frac{1}{\Delta_{c} f}\right) \\
& \leq m\left(r, \frac{1}{\Delta_{c} f}\right)+S(r, f) .
\end{aligned}
$$

By the first fundamental theorem of Nevanlinna theory and (13), we can get

$$
T(r, f) \leq T\left(r, \Delta_{c} f\right)+N\left(r, \frac{1}{f}\right)+S(r, f) .
$$

Hence

$$
\begin{aligned}
1 & \leq \liminf _{r \rightarrow \infty} \frac{T\left(r, \Delta_{c} f\right)}{T(r, f)}+\limsup _{r \rightarrow \infty} \frac{N\left(r, \frac{1}{f}\right)}{T(r, f)} \\
& =\liminf _{r \rightarrow \infty} \frac{T\left(r, \Delta_{c} f\right)}{T(r, f)}+(1-\delta(0, f)) \\
& =\liminf _{r \rightarrow \infty} \frac{T\left(r, \Delta_{c} f\right)}{T(r, f)} .
\end{aligned}
$$


On the other hand, we have

$$
\begin{aligned}
T\left(r, \Delta_{c} f\right) & =m\left(r, \Delta_{c} f\right)+N\left(r, \Delta_{c} f\right) \\
& =m\left(r, \frac{f \Delta_{c} f}{f}\right)+N\left(r, \Delta_{f} f\right) \\
& \leq m\left(r, \frac{\Delta_{c} f}{f}\right)+m(r, f)+N(r, f)+N(r, f(z+c)) .
\end{aligned}
$$

It follows from Lemma 2.2 and Lemma 2.5 that

$$
T\left(r, \Delta_{c} f\right) \leq T(r, f)+N(r, f)+S(r, f) .
$$

As $\delta(\infty, f)=1$, so

$$
\limsup _{r \rightarrow \infty} \frac{T\left(r, \Delta_{c} f\right)}{T(r, f)} \leq 1+\limsup _{r \rightarrow \infty} \frac{N(r, f)}{T(r, f)}=1 .
$$

Therefore

$$
\lim _{r \rightarrow+\infty} \frac{T\left(r, \Delta_{c} f\right)}{T(r, f)}=1 .
$$

Since $\delta(0, f)=1$ and $\delta(\infty, f)=1$, then

$$
N\left(r, \frac{1}{f}\right)=S(r, f), N(r, f)=S(r, f) .
$$

By (17) and Lemma 2.6, we have

$$
\begin{aligned}
T(r, f) & \leq N\left(r, \frac{1}{\left(\Delta_{c} f\right)-z}\right)+S(r, f) \\
& \leq T\left(r, \frac{1}{\left(\Delta_{c} f\right)-z}\right)+S(r, f) \\
& \leq T\left(r, \Delta_{c} f\right)+S(r, f) .
\end{aligned}
$$

Combining (16) and (18) implies

$$
T\left(r, \Delta_{c} f\right) \sim T(r, f) \sim N\left(r, \frac{1}{\left(\Delta_{c} f\right)-z}\right),
$$

as $r \rightarrow \infty$. 
Authors' contributions

All authors drafted the manuscript, read and approved the final manuscript. All authors contributed equally.

\section{Author details}

'School of Mathematics and Statistics, Hubei University of Science and Technology, Xianning, China. ${ }^{2}$ Hubei Key Laboratory of Applied Mathematics, Faculty of Mathematics and Statistics, Hubei University, Wuhan, China. ${ }^{3}$ Xianning Vocational Technical College, Xianning, China.

\section{Publisher's Note}

Springer Nature remains neutral with regard to jurisdictional claims in published maps and institutional affiliations.

\section{Received: 29 May 2019 Accepted: 17 October 2019 Published online: 28 October 2019}

\section{References}

1. Bergweiler, W., Langley, J.K.: Zeros of differences of meromorphic functions. Math. Proc. Camb. Philos. Soc. 142, 133-147 (2007)

2. Chen, Z.X.: The fixed points and hyper order of solutions of second order complex differential equations. Acta Math. Sci. Ser. A 20(3), 425-432 (2000)

3. Chen, Z.X.: Fixed points of meromorphic functions and of their difference and shifts. Ann. Pol. Math. 109(2), 153-163 (2013)

4. Chen, Z.X., Shon, K.H.: Fixed points of meromorphic solutions for some difference equations. Abstr. Appl. Anal. 2013, Article ID 496096 (2013)

5. Chiang, Y.M., Feng, S.J.: On the Nevanlinna characteristic of $f(z+\eta)$ and difference equations in the complex plane. Ramanujan J. 16, 105-129 (2008)

6. Chiang, Y.M., Feng, S.J.: On the growth of logarithmic difference, difference equations and logarithmic derivatives of meromorphic functions. Transl. Am. Math. Soc. 361, 3767-3791 (2009)

7. Chuang, C.T., Yang, C.C.: Theory of Fix Points and Factorization of Meromorphic Functions. Mathematical Monograph Series. Peking University Press, Beijing (1986)

8. Cui, W.W., Yang, L.Z.: Zeros and fixed points of difference operators of meromorphic functions. Acta Math. Sci. 33B(3), 773-780 (2013)

9. Halburd, R.G., Korhonen, R.J.: Nevanlinna theory for the difference operator. Ann. Acad. Sci. Fenn., Math. 31, 463-478 (2006)

10. Halburd, R.G., Korhonen, R.J.: Difference analogue of the lemma on the logarithmic derivative with applications to difference equations. J. Math. Anal. Appl. 314, 477-487 (2006)

11. Halburd, R.G., Korhonen, R.J.: Meromorphic solutions of difference equations, integrability and the discrete Painlev'e equations. J. Phys. A, Math. Theor. 40, 1-38 (2007)

12. Hayman, W.K.: Meromorphic Functions. Oxford Mathematical Monographs. Clarendon, Oxford (1964)

13. Lahiri, I.: Milloux theorem, deficiency and fix-points for vector-valued meromorphic functions. J. Indian Math. Soc. 59, 45-60 (1993)

14. Li, N., Yang, L.Z.: Value distribution of difference and q-difference polynomials. Adv. Differ. Equ. 2013, 98, 1-9 (2013)

15. Liu, K., Cao, H.Z., Cao, T.B.: Entire solutions of Fermat type differential difference equations. Arch. Math. 99, 147-155 (2012)

16. Wu, Z.J.: Value distribution for difference operator of meromorphic functions with maximal deficiency sum. J. Inequal. Appl. 2013, 530, 1-9 (2013)

17. Xu, H.Y., Cao, T.B., Liu, B.X.: The growth of solutions of systems of complex q-shift difference equations. Adv. Differ. Equ. $2012,216,1-22(2012)$

18. Xu, J.F., Zhang, X.B.: The zeros of q-shift difference polynomials of meromorphic functions. Adv. Differ. Equ. 2012, 200, $1-10(2012)$

19. Yang, C.C., Yi, H.X.: Uniqueness Theory of Meromorphic Functions. Mathematics and Its Application, vol. 557. Kluwer Academic, Dordrecht (2003)

20. Yang, L.: Value Distribution Theory. Springer, Berlin (1993) Translated and revised from the 1982 Chinese original

21. Zhang, R.R., Chen, Z.X.: Value distribution of difference polynomials of meromorphic functions. Sci. Sin., Math. 42(11), $1115-1130$ (2012) (in Chinese)

22. Zhang, R.R., Chen, Z.X.: Fixed points of meromorphic functions and of their difference, divided differences and shifts Acta Math. Sin. New Ser. 32(10), 1189-1202 (2016)

23. Zheng, J.H.: Value Distribution of Meromorphic Functions. Tsinghua University Press, Beijing (2010) 